Bull. Korean Math. Soc. 49 (2012), No. 2, pp. 367-372

http://dx.doi.org/10.4134/BKMS.2012.49.2.367

\title{
ON THE ERROR TERM IN THE PRIME GEODESIC THEOREM
}

\author{
Muharem Avdispahić And DžEnan Gušić
}

\begin{abstract}
Taking the integrated Chebyshev-type counting function of the appropriate order, we improve the error term in Park's prime geodesic theorem for hyperbolic manifolds with cusps. The obtained estimate coincides with the best known result in the Riemann surfaces case.
\end{abstract}

Let $\Gamma$ be a discrete co-finite torsion free subgroup of $G=\mathrm{SO}_{0}(d, 1)$ satisfying the condition $\Gamma \cap P=\Gamma \cap N(P)$ for $P \in \mathfrak{P}_{\Gamma}$, where $\mathfrak{P}_{\Gamma}$ is the set of $\Gamma$ conjugacy classes of $\Gamma$-cuspidal parabolic subgroups in $G$ and $N(P)$ is the unipotent part of $P$. Denote by $K$ a maximal compact subgroup of $G$. The manifold $X_{\Gamma}=\Gamma \backslash G / K$ is a $d$-dimensional real hyperbolic manifold with cusps.

As usual, let $\pi_{\Gamma}(x)$ be the number of prime geodesics $C_{\gamma}$ of length $l_{\gamma} \leq \log x$ on $X_{\Gamma}$. Recall that prime geodesic $C_{\gamma}$ corresponds to the conjugacy class $\gamma$ of a primitive hyperbolic element with the norm $N(\gamma)=e^{l_{\gamma}}$.

The purpose of this short note is to prove that Park's refinement of the prime geodesic theorem, due to Gangolli [5] and DeGeorge [3] in the compact case and to Gangolli-Warner [6] in the finite volume case, can be further improved to obtain the theorem in the following form.

Theorem 1. Let $X_{\Gamma}$ be as above. Then

$$
\pi_{\Gamma}(x)=\sum_{\frac{3}{2} d_{0}<s_{n}(k) \leq 2 d_{0}}(-1)^{k} \operatorname{li}\left(x^{s_{n}(k)}\right)+O\left(x^{\frac{3}{2} d_{0}}(\log x)^{-1}\right)
$$

as $x \rightarrow+\infty$, where $d_{0}=\frac{d-1}{2},\left(s_{n}(k)-k\right)\left(2 d_{0}-k-s_{n}(k)\right)$ is a small eigenvalue in $\left[0, \frac{3}{4} d_{0}^{2}\right]$ of $\Delta_{k}$ on $\pi_{\sigma_{k}, \lambda_{n}(k)}$ with $s_{n}(k)=d_{0}+i \lambda_{n}(k)$ or $s_{n}(k)=$ $d_{0}-i \lambda_{n}(k)$ in $\left(\frac{3}{2} d_{0}, 2 d_{0}\right], \Delta_{k}$ is the Laplacian acting on the space of $k$-forms over $X_{\Gamma}$ and $\pi_{\sigma_{k}, \lambda_{n}(k)}$ is the principal series representation.

Proof. Let $\Gamma_{\mathrm{h}}$ (resp. $\mathrm{P} \Gamma_{\mathrm{h}}$ ) denote the set of the $\Gamma$-conjugacy classes of hyperbolic (resp. primitive hyperbolic) elements in $\Gamma$. Set $\Lambda(\gamma)=l_{\gamma_{0}}$, where

Received November 5, 2010; Revised April 14, 2011.

2010 Mathematics Subject Classification. 11M36, 11F72.

Key words and phrases. Ruelle zeta function, prime geodesic theorem.

This work has been partially supported by a grant from the Federal Ministry of Science and Education of Bosnia and Herzegovina. 
$\gamma=\gamma_{0}^{j(\gamma)}, \gamma_{0} \in \mathrm{P} \Gamma_{\mathrm{h}}, j(\gamma) \in \mathbb{N}$. It is well known that the assertion of the theorem can be easily deduced from the relation

(1) $\quad \psi_{0}(x)=\sum_{\gamma \in \Gamma_{\mathrm{h}}, N(\gamma) \leq x} \Lambda(\gamma)=\sum_{s_{n}(k) \in\left(\frac{3}{2} d_{0}, 2 d_{0}\right]} \frac{(-1)^{k} x^{s_{n}(k)}}{s_{n}(k)}+O\left(x^{\frac{3}{2} d_{0}}\right)$.

We observe that J. Park [12] proved a variant of (1) with the error term $O\left(x^{\frac{3}{2} d_{0}}(\log x)^{\frac{1}{2}}\right)$ in place of $O\left(x^{\frac{3}{2} d_{0}}\right)$. The key role in his proof is played by the Ruelle zeta function replacing the Selberg zeta. This is in line with Parry-Pollicott [13]. The ingredients come from the results of Fried [4] and further investigations of the Ruelle zeta by Gon-Park [7]. The error term $O\left(x^{\frac{3}{2} d_{0}}(\log x)^{\frac{1}{2}}\right)$ stems from Park's modification of Hejhal's techniques [8], [9] and the use of a higher order counting function $\psi_{d-1}(x)$, where $\psi_{n}(x)$ is defined recursively by $\psi_{n}(x)=\int_{0}^{x} \psi_{n-1}(t) d t$ for $n \in \mathbb{N}$. Inspired by Randol's approach in the case of compact Riemann surfaces, we consider $\psi_{d}(x)$ instead of $\psi_{d-1}(x)$.

It is well known that $\psi_{d}(x)$ can be represented in the form

$$
\psi_{d}(x)=-\frac{1}{2 \pi i} \int_{c-i \infty}^{c+i \infty} \frac{R_{\Gamma}^{\prime}(s)}{R_{\Gamma}(s)} \frac{x^{s+d}}{s(s+1) \cdots(s+d)} d s
$$

for some $c>2 d_{0}$. Here, $R_{\Gamma}$ is the Ruelle zeta function defined by

$$
R_{\Gamma}(s)=\prod_{\gamma_{0} \in \mathrm{P} \Gamma_{\mathrm{h}}}\left(1-e^{-s l_{\gamma_{0}}}\right)^{-1}, \operatorname{Re}(s)>2 d_{0},
$$

and meromorphically continued to the whole complex plane. Following [12], we apply the Cauchy residue theorem to the integrand of $\psi_{d}(x)$ over $R(T)=$ $\left\{s \in \mathbb{C}|| s \mid \leq T, \operatorname{Re}(s) \leq d_{0}\right\} \cup\left\{s \in \mathbb{C} \mid d_{0} \leq \operatorname{Re}(s) \leq c,-\widetilde{T} \leq \operatorname{Im}(s) \leq \widetilde{T}\right\}$, where $\widetilde{T}=\sqrt{T^{2}-d_{0}^{2}}$ and $T \gg 0$ is such that there is no zero or pole of the integrand over the boundary of $R(T)$.

For a fixed $0<\varepsilon<c-d_{0}$, the adjusted Park argumentation gives us

$$
\begin{aligned}
& \frac{1}{2 \pi i} \int_{c-i \widetilde{T}}^{c+i \widetilde{T}} \frac{R_{\Gamma}^{\prime}(s)}{R_{\Gamma}(s)} \frac{x^{s+d}}{s(s+1) \cdots(s+d)} d s \\
= & -\frac{1}{2 \pi i} \int_{C_{T}}+\frac{1}{2 \pi i}\left(\int_{d_{0}+i \widetilde{T}}^{d_{0}+\varepsilon+i \widetilde{T}}+\int_{d_{0}+\varepsilon-i \widetilde{T}}^{d_{0}-i \widetilde{T}}\right)+\frac{1}{2 \pi i}\left(\int_{d_{0}+\varepsilon+i \widetilde{T}}^{c+i \widetilde{T}}+\int_{c-i \widetilde{T}}^{d_{0}+\varepsilon-i \widetilde{T}}\right) \\
& +\sum_{z \in R(T)} \operatorname{Res}_{s=z}\left(\frac{R_{\Gamma}^{\prime}(s)}{R_{\Gamma}(s)} \frac{x^{s+d}}{s(s+1) \cdots(s+d)}\right) \\
= & -\frac{1}{2 \pi i} \int_{C_{T}}+O\left(x^{d+d_{0}+\varepsilon} T^{-2}\right)+O\left(\varepsilon^{-1} x^{c+d} T^{-2}\right)
\end{aligned}
$$




$$
+\sum_{z \in R(T)} \operatorname{Res}_{s=z}\left(\frac{R_{\Gamma}^{\prime}(s)}{R_{\Gamma}(s)} \frac{x^{s+d}}{s(s+1) \cdots(s+d)}\right),
$$

where $C_{T}$ is the anti-clockwise oriented circular part of the boundary of $R(T)$.

Now, the first integral on the right hand side of (2) can be estimated by

$$
\frac{1}{2 \pi i} \int_{C_{T}} \frac{R_{\Gamma}^{\prime}(s)}{R_{\Gamma}(s)} \frac{x^{s+d}}{s(s+1) \cdots(s+d)} d s=O\left(x^{d+d_{0}} T^{-1} \log T\right),
$$

since

$$
\begin{aligned}
\left|\frac{1}{2 \pi i} \int_{C_{T}} \frac{R_{\Gamma}^{\prime}(s)}{R_{\Gamma}(s)} \frac{x^{s+d}}{s(s+1) \cdots(s+d)} d s\right| & \leq C_{1} x^{d+d_{0}} T^{-(d+1)} \int_{C_{T}}\left|\frac{R_{\Gamma}^{\prime}(s)}{R_{\Gamma}(s)}\right||d s| \\
& \leq C_{1} x^{d+d_{0}} T^{-(d+1)} \int_{|s|=T}\left|\frac{R_{\Gamma}^{\prime}(s)}{R_{\Gamma}(s)}\right||d s| \\
& \leq C_{2} x^{d+d_{0}} T^{-1} \log T
\end{aligned}
$$

according to [4, p. 509, Prop. 7].

We note that (3) is to be compared to [12, relation (3.8)].

Furthermore,

$$
\begin{aligned}
& \sum_{z \in R(T)} \operatorname{Res}_{s=z}\left(-\frac{R_{\Gamma}^{\prime}(s)}{R_{\Gamma}(s)} \frac{x^{s+d}}{s(s+1) \cdots(s+d)}\right) \\
= & \sum_{s_{n}(k) \in\left(d_{0}, 2 d_{0}\right]}(-1)^{k} \frac{x^{s_{n}(k)+d}}{s_{n}(k)\left(s_{n}(k)+1\right) \cdots\left(s_{n}(k)+d\right)} \\
& +\sum_{-\widetilde{T} \leq \lambda_{n}(0) \leq \widetilde{T}} \frac{x^{s_{n}(0)+d}}{s_{n}(0)\left(s_{n}(0)+1\right) \cdots\left(s_{n}(0)+d\right)} \\
& +\sum_{z \in R\left(T, d_{0}\right)} \operatorname{Res}_{s=z}\left(-\frac{R_{\Gamma}^{\prime}(s)}{R_{\Gamma}(s)} \frac{x^{s+d}}{s(s+1) \cdots(s+d)}\right),
\end{aligned}
$$

where $R\left(T, d_{0}\right)=R(T) \cap\left\{s \in \mathbb{C} \mid \operatorname{Re}(s)<d_{0}\right\}$.

However,

$$
\sum_{z \in R\left(T, d_{0}\right)} \operatorname{Res}_{s=z}\left(-\frac{R_{\Gamma}^{\prime}(s)}{R_{\Gamma}(s)} \frac{x^{s+d}}{s(s+1) \cdots(s+d)}\right)=O\left(x^{d+d_{0}} T^{-1} \log T\right),
$$

as opposed to $[12,(3.17)]$.

Taking into account (3), (4), (5), as well as the fact that

$$
\frac{1}{2 \pi i} \int_{c-i \widetilde{T}}^{c+i \widetilde{T}} \frac{R_{\Gamma}^{\prime}(s)}{R_{\Gamma}(s)} \frac{x^{s+d}}{s(s+1) \cdots(s+d)} d s=-\psi_{d}(x)+O\left(x^{c+d} T^{-d}\right)
$$


and passing to the limit $T \rightarrow+\infty$ in (2), we end up with

$$
\begin{aligned}
\psi_{d}(x)= & \sum_{s_{n}(k) \in\left(d_{0}, 2 d_{0}\right]}(-1)^{k} \frac{x^{s_{n}(k)+d}}{s_{n}(k)\left(s_{n}(k)+1\right) \cdots\left(s_{n}(k)+d\right)} \\
& +\sum_{s_{n}(0)=d_{0} \pm i \lambda_{n}(0)} \frac{x^{s_{n}(0)+d}}{s_{n}(0)\left(s_{n}(0)+1\right) \cdots\left(s_{n}(0)+d\right)} .
\end{aligned}
$$

To derive the asymptotics of $\psi_{0}(x)$ from the asymptotics of $\psi_{d}(x)$, one proceeds in the standard way introducing the functions

$$
\Delta_{d}^{+} f(x)=\int_{x}^{x+h} \int_{x_{2 d_{0}}}^{x_{2 d_{0}}+h} \cdots \int_{x_{1}}^{x_{1}+h} f^{(d)}\left(x_{0}\right) d x_{0} \cdots d x_{2 d_{0}}
$$

and

$$
\Delta_{d}^{-} f(x)=\int_{x-h}^{x} \int_{x_{2 d_{0}-h}}^{x_{2 d_{0}}} \cdots \int_{x_{1}-h}^{x_{1}} f^{(d)}\left(x_{0}\right) d x_{0} \cdots d x_{2 d_{0}}
$$

for some constant $h$ to be specified later on.

Notice that

$$
h^{-d} \Delta_{d}^{+} \frac{x^{s_{n}(k)+d}}{s_{n}(k)\left(s_{n}(k)+1\right) \cdots\left(s_{n}(k)+d\right)}=\frac{x^{s_{n}(k)}}{s_{n}(k)}+O\left(h^{s_{n}(k)}\right)
$$

and

$$
\begin{aligned}
& h^{-d} \Delta_{d}^{+} \frac{x^{s_{n}(0)+d}}{s_{n}(0)\left(s_{n}(0)+1\right) \cdots\left(s_{n}(0)+d\right)} \\
= & O\left(\min \left(x^{d_{0}}\left|s_{n}(0)\right|^{-1}, h^{-d}\left|s_{n}(0)\right|^{-(d+1)} x^{d+d_{0}}\right)\right) .
\end{aligned}
$$

The second bound in (8) is straightforward from the representation

$$
\Delta_{d}^{+} f(x)=\sum_{i=0}^{d}(-1)^{i}\left(\begin{array}{l}
d \\
i
\end{array}\right) f(x+(d-i) h) .
$$

The relation (7) and the first bound in (8) are obtained by application of the mean value theorem (Cf. [8, p. 114]), [14, p. 246]).

Following [11, pp. 463-464] and using (8) we deduce

$$
\begin{aligned}
& h^{-d} \Delta_{d}^{+} \sum_{s_{n}(0)=d_{0} \pm i \lambda_{n}(0)} \frac{x^{s_{n}(0)+d}}{s_{n}(0)\left(s_{n}(0)+1\right) \cdots\left(s_{n}(0)+d\right)} \\
= & O\left(x^{d_{0}} \int_{d_{0}}^{M} t^{-1} d N(t)\right)+O\left(h^{-d} x^{d+d_{0}} \int_{M}^{+\infty} t^{-(d+1)} d N(t)\right) \\
= & O\left(x^{d_{0}} M^{2 d_{0}}\right)+O\left(h^{-d} x^{d+d_{0}} M^{-1}\right)
\end{aligned}
$$


for some $M>2 d_{0}$, where $N(t)=O\left(t^{d}\right)$ denotes the counting function of $s_{n}(0)=d_{0}+i \lambda_{n}(0)$. Thus, (6), (7) and (9) imply

$$
\begin{aligned}
h^{-d} \Delta_{d}^{+} \psi_{d}(x)= & \sum_{s_{n}(k) \in\left(d_{0}, 2 d_{0}\right]} \frac{(-1)^{k} x^{s_{n}(k)}}{s_{n}(k)}+O\left(h^{2 d_{0}}\right) \\
& +O\left(x^{d_{0}} M^{2 d_{0}}\right)+O\left(h^{-d} x^{d+d_{0}} M^{-1}\right) .
\end{aligned}
$$

Substituting $M=x^{\frac{1}{4}}, h=x^{\frac{3}{4}}$ into (10), we get

$$
\psi_{0}(x) \leq h^{-d} \Delta_{d}^{+} \psi_{d}(x)=\sum_{s_{n}(k) \in\left(\frac{3}{2} d_{0}, 2 d_{0}\right]} \frac{(-1)^{k} x^{s_{n}(k)}}{s_{n}(k)}+O\left(x^{\frac{3}{2} d_{0}}\right) .
$$

Reasoning in an analogous way, one proves

$$
\sum_{s_{n}(k) \in\left(\frac{3}{2} d_{0}, 2 d_{0}\right]} \frac{(-1)^{k} x^{s_{n}(k)}}{s_{n}(k)}+O\left(x^{\frac{3}{2} d_{0}}\right)=h^{-d} \Delta_{d}^{-} \psi_{d}(x) \leq \psi_{0}(x) .
$$

Combining (11) and (12), we finally obtain

$$
\psi_{0}(x)=\sum_{s_{n}(k) \in\left(\frac{3}{2} d_{0}, 2 d_{0}\right]} \frac{(-1)^{k} x^{s_{n}(k)}}{s_{n}(k)}+O\left(x^{\frac{3}{2} d_{0}}\right) .
$$

This completes the proof.

Final Remarks. It is easily seen that taking $\psi_{n}(x)$ with $n>d$ does not yield a better result. The obtained error term $O\left(x^{\frac{3}{2} d_{0}}(\log x)^{-1}\right)$ in the prime geodesic theorem is in accordance with the known estimate in the case of Riemann surfaces that can be achieved through several different approaches (see, e.g., [1], [2], [14]). Actually, in the Concluding Remark of [14], Randol noted that it would be interesting to determine the extent to which his methods are applicable to more general symmetric spaces. Theorem 1 can be interpreted as an answer to this query in our setting.

Acknowledgment. We would like to thank the referee for the valuable suggestions that improved the presentation.

\section{References}

[1] M. Avdispahić and L. Smajlović, On the prime number theorem for a compact Riemmann surface, Rocky Mountain J. Math. 39 (2009), no. 6, 1837-1845.

[2] P. Buser, Geometry and Spectra of Compact Riemann Surfaces, Progress in Mathematics, Vol. 106 Birkhäuser, Boston-Basel-Berlin, 1992.

[3] D. L. DeGeorge, Length spectrum for compact locally symmetric spaces of strictly negative curvature, Ann. Sci. École Norm. Sup. (4) 10 (1977), no. 2, 133-152.

[4] D. Fried, The zeta functions of Ruelle and Selberg, Ann. Sci. École Norm. Sup. (4) 19 (1986), no. 4, 491-517.

[5] R. Gangolli, The length spectrum of some compact manifolds of negative curvature, J. Differential Geom. 12 (1977), no. 3, 403-424. 
[6] R. Gangolli and G. Warner, Zeta functions of Selberg's type for some noncompact quotients of symmetric spaces of rank one, Nagoya Math. J. 78 (1980), 1-44.

[7] Y. Gon and J. Park, The zeta functions of Ruelle and Selberg for hyperbolic manifolds with cusps, Math. Ann. 346 (2010), no. 3, 719-767.

[8] D. Hejhal, The Selberg Trace Formula for PSL $(2, \mathbb{R})$. Vol. I, Lecture Notes in Mathematics 548. Springer-Verlag, Berlin-Heidelberg, 1973.

[9] — The Selberg Trace Formula for PSL $(2, \mathbb{R})$. Vol. II, Lecture Notes in Mathematics 1001. Springer-Verlag, Berlin-Heidelberg, 1983.

[10] H. Huber, Zur analytischen Theorie hyperbolischer Raumformen und Bewegungsgrupen II, Math. Ann. 142 (1961), 385-398.

[11] _ Nachtrag zu [10], Math. Ann. 143 (1961), 463-464.

[12] J. Park, Ruelle zeta function and prime geodesic theorem for hyperbolic manifolds with cusps, in: G. van Dijk, M. Wakayama (eds.), Casimir Force, Casimir Operators and the Riemann Hypothesis, 9-13 November 2009, Kyushu University, Fukuoka, Japan, Walter de Gruyter, 2010.

[13] W. Parry and M. Pollicott, An analogue of the prime number theorem for closed orbits of Axiom A flows, Ann. of Math. (2) 118 (1983), no. 3, 573-591.

[14] B. Randol, On the asymptotic distribution of closed geodesics on compact Riemann surfaces, Trans. Amer. Math. Soc. 233 (1977), 241-247.

Muharem Avdispahić

Department of Mathematics

UNIVERSITY OF SARAJEVO

Zmaja od Bosne 35, 71000 Sarajevo, Bosnia and Herzegovina

E-mail address: mavdispa@pmf.unsa.ba

DžENAN GuŠIĆ

Department of Mathematics

UNIVERSity OF SARAJEVO

Zmaja od Bosne 35,71000 Sarajevo, Bosnia and Herzegovina

E-mail address: dzenang@yahoo.com 